\title{
Efficacy of sound treatment for better meditation and sleep in music therapy
}

\begin{abstract}
Music therapy (MT) has contributed health promotion of body and mind. The authors have continued healing video/music development mainly for $4 \mathrm{~K}$ quality video, and delivered high-quality natural environment scene/music video distribution service collaborated by the first-class company. The concept and theme of the activities are "sound treatment every day" and "change your meditation and sleep". The company name is Croix from French, which stands for the cross in English with the meaning of healing, protection, supporting each other. Providing adequate mindfulness and lifestyle-related music brings the improvement of Subjective Happiness Scale (SHS) and the reduction of stress-related problems.
\end{abstract}

Keywords: Integrative medicine Japan, music therapy, sound treatment every day, change your meditation and sleep, subjective happiness scale, Croix
Volume 14 Issue 4 - 202 I

\author{
Bajune Tobeta,' Hiroshi Bando,, 2 \\ 'CEO \& President, Croix Co. Ltd., Japan \\ ${ }^{2}$ Medical Research/Tokushima University, Japan \\ ${ }^{3}$ Integrative Medicine Japan, Shikoku Island Division, Japan
}

Correspondence: Hiroshi Bando, Tokushima University / Medical Research, Nakashowa I-6I,Tokushima 770-0943 Japan, Tel +8I-90-3 I87-2485, Email pianomed@bronze.ocn.ne.jp

Received: June 30, 202I | Published: August 02, 2021
Abbreviations: IM, integrative medicine; QOL, quality of life; CAM, complementary and alternative medicine; WM, western medicine; SHS, subjective happiness scale; SWB, subjective wellbeing

\section{Commentary}

Medicine and music have been closely related since ancient times. ${ }^{1}$ As religion is added to these, three fundamental factors are shown for human beings. Music therapy has long roots and has been effective since ancient civilization. ${ }^{2}$ Aristotle found a clear medical value in music. There is also a historical fact that doctor Asclepius actually recommended music to people with emotional problems. ${ }^{3}$ The Old Testament states that "David cured Saul's depression with musical instrument lyre," and the Egyptians regarded music as "soul meds." The Persians are said to have been cured of their illness by music with a lute (a stringed instrument similar to a guitar).

In recent decade, music therapy has been widely understood and accepted in integrative medicine (IM), which includes all region of medical treatments. ${ }^{4}$ Its functions aim to recover from physical and mental disorders, improve quality of life (QOL), relieve stress, and boost immunity by using the physiological, psychological and social functions of music. Music is included in the category of art and culture, and music therapy is also included in the category of art therapy. 5

In other words, it is involved in hearing and singing, as well as all five senses such as sight, smell, taste and touch. As IM covers complementary and alternative medicine (CAM) and Western Medicine (WM), music therapy covers not only the mind/body, but also the bio-psycho-social aspect. Consequently, music mainly includes some factors of hearing and vision. On the other hand, music therapy may include the combination of five senses of human being and somewhat the sixth sense such as inspiration and telepathy of the related people around. ${ }^{6}$

Authors' team of music therapy have continued clinical practice and research with physicians, music therapists and specialists, and it is CROIX for health and wellness that has expanded music therapy so far. ${ }^{7,8}$ Sound treatments have been shown to be effective, which mean various health methods related to music. Several examples are found and presented worldwide, such as Mozart and Bach, sleeping music, relaxation music, meditation music, healing natural sounds, yoga music, Zen music and so on. ${ }^{9,10}$ Our fundamental philosophy is summarized as "Sound Treatment Everyday" (Figure 1).

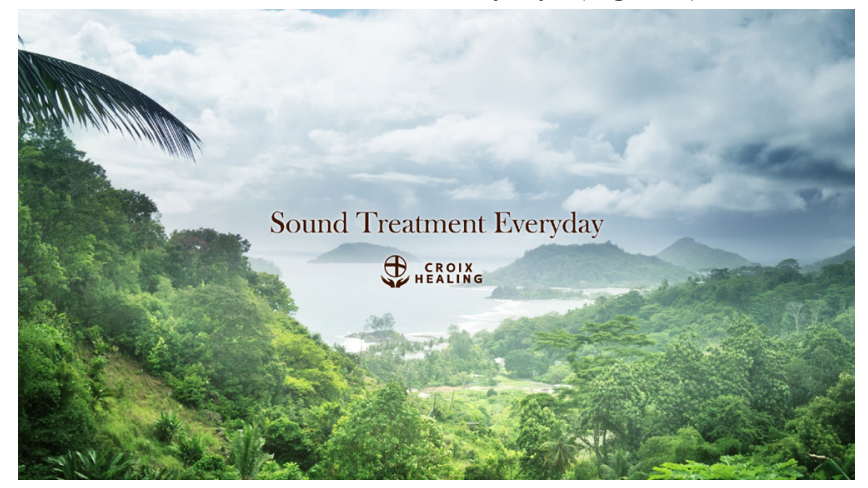

Figure I The Concept of Croix: Sound Treatment Everyday.

The name Croix comes from the French meaning of cross in English. The word cross has several meanings. As Red Cross hospitals in the world use the logo mark of Red Cross, it means medical care or cutting a cross. ${ }^{11}$ It includes the meaning of healing and protection. It also has the deep meaning of supporting each other. For the reason of the choice for French language, the historical fact was observed that the founder Tobeta was greatly influenced by French Impressionist classical music.

Regarding the background of the author, previous profession included originally a music composer, lyricist and producer. The original healing sound world was established through music activities and releasing various co-starring works with some of the best musician's worldwide. ${ }^{7}$ As a basis for these experiences, the company Croix was established in 2009 and become a member of Japanese Recording Industry Association. Since then, it has grown to a topclass market share in the Japanese healing music field and domestic music market, and has contributed to people's physical and mental health.

During the long life of each person, one engages in various activities during the day and rests at night. It is the essential physiological activity 
and has a regular rhythm. It would be great if the sleep is brought smoothly associated with good dreams. A good night's sleep requires a calm state of mind at night, and ideally a quiet meditation time. ${ }^{12}$ In this way, our staffs hope to deliver ideal meditation and sleep to many people across the world. ${ }^{13}$ Thus, our corporate philosophy and/ or slogan would be "Change Your Meditation and Sleep". We believe that continuing our research and practice will be sure to transform the world providing good meditation and sleep. ${ }^{9}$

We have concrete means to change the "sleep" of the world better. They include i) Sound/video content and subscription services which are developed in collaboration with doctors of medicine and specialized agencies, ii) Various content to promote quality meditation and mental stability, iii) Beautifully designed and produced functional trans-technical services, iv) YouTube channel released from CROIX HEALING. ${ }^{14}$ v) Healing video/music development mainly for 4K quality video from 2019, vi) Delivering high-quality natural environment scene and music video distribution service collaborated by the first-class domestic company.

There are two aspects for listening and recognizing music. One is the auditory efficacy of analyzing sound in the air, where we can hear the sound by the electric signal from ear to cerebrum. ${ }^{15}$ Another is the psychological efficacy of adapting the presence of music, where we can feel and tune the rhythm of music. In our human society, adequate adaptation to these two mechanisms can bring the cultural evolution of music. Furthermore, it will produce the diversity of music forms and behaviors across the world.

The study of Subjective Happiness Scale (SHS) has been known and conducted. ${ }^{16}$ The important region is the anterior right wedge (precuneus) in the brain. ${ }^{17}$ This area has the role of sensory information, and this increased activity leads to negative self-efficacy and losing mind. When the people are in the meditation, the precuneus remained suppressed associated with reduced confusion and elevated stability. ${ }^{17}$

From a variety of research, medical data have been revealed by usually measurable biomarkers. However, several certain impact factors are described as emotional status and subjective well-being (SWB). ${ }^{18}$ Happiness has been explained from psychological point of view. Among them, several factors are included such as negative emotion, positive happiness, daily time management and various psychological interventions. ${ }^{19}$

As to listening to various kinds of music, do we recall certain common feeling associated with some evidence of universality? A recent study showed 13 types of subjective feelings evoked by listening to 2168 different music excerpts. ${ }^{20}$ These types are shown as follows: Amusing, Annoying, Anxious-tense, Beautiful, Calm-relaxing serene, Dreamy, Energizing-pump-up, Erotic-desirous, Indignant-defiant, Joyful-cheerful, Sad-depressing, Scary-fearful, Triumphant-heroic. As mentioned above, various subjective feelings are evoked by music, and in contrast, music can make changing emotion stable by adequate presentation of music.

For some decades, music has been used for providing relaxation and calmness. ${ }^{21}$ Consequently, the interventions of music therapy have been evaluated to reduce stressful matter and increase the well-being of subjects for various populations. ${ }^{22}$ In order to assess the influence of stress, it is important to evaluate the efficacy of non-pharmacological therapeutic interventions such as music therapy. Large studies on the relationship between music listening and psychological stress-related outcomes were conducted. ${ }^{23}$ It included 2,747 cases from 47 studies and 76 effect sizes. As a result, music therapy brought medium-large efficacy on stress-related outcomes (mean effect size, $d=0.723,95 \%$ Cl 0.51-0.94, $\mathrm{p}<0.001)$.

In the fields of health, music and technology, significant interactions have been recently observed for developing technology of music and well-being. ${ }^{24}$ Several workshops of music, health and computing have been conducted in order to strengthen the mutual collaboration among health, medicine, music and technology. Meaningful discussion has continued concerning psychology, neuroscience, medical technology, music information, music therapy, and robotics. ${ }^{24}$ From all the situations and perspectives mentioned above, it is expected that the cooperation between music and medicine will contribute to the wellbeing of people in the future..$^{25}$

\section{Acknowledgments}

None.

\section{Conflicts of interest}

The authors declare no conflict of interest.

\section{Funding}

There was no funding received for this paper.

\section{References}

1. Fiore J. Randomized pilot study exploring an online pre-composed receptive music experience and a mindfulness-based intervention for hospice workers' stress and professional quality of life. The Arts in Psychotherapy. 2021;74:101797.

2. Bando H. Music Therapy. Singapore: Raffles Connect PTE; 2020.

3. Hirai Y, Bando H, Yoshioka A, et al. Music and Man in Art: The Future of Media and Technology. Global J Arts Social Sci. 2020;2(1):116.

4. Moore C, Elliott D. Introduction to music therapy practice. $J$ Music Ther. 2019;56(1):117-121.

5. Bando H, Yoshioka A, Nishikiori Y. Various Care Option of Integrative Medicine from the Viewpoint of Patient-Oriented Medicine. Int J Conf Proc. 2020;2(1). ICP. 000529.2020.

6. Warth M, Koehler F, Weber M, et al. Song of Life (SOL) study protocol: a multicenter, randomized trial on the emotional, spiritual, and psychobiological effects of music therapy in palliative care. $B M C$ Palliative Care. 2019;18(1):14.

7. Croix Co. Ltd, Tokyo, Japan.

8. Bando H, Yoshioka A, Nishikiori Y. Future research direction from the perspective of music therapy. Art Human Open Acc J. 2020;4(2):54-56.

9. Mindfulness-related label. CROIX Healing.

10. Lifestyle-related label. Sugar Candy.

11. Wylie N, Oppenheimer M, Crossland J. The Red Cross Movement Myths, practices, turning points. England: Manchester University Press; 2021.

12. Shrivastava A, Singh BK, Nirala N. Chapter 4. A study of the influence of meditation and music therapy on the vital parameters of the human body through EEG signal analysis: a review. IOP Publishing Ltd. 2020:1-17.

13. Nanthakwang N, Siviroj P, Matanasarawoot A, et al. Effectiveness of Deep Breathing and Body Scan Meditation Combined with Music to Improve Sleep Quality and Quality of Life in Older Adults. The Open Public Health Journal. 2020;13:232-239.

14. https://www.youtube.com/c/CROIXHEALING 
15. Mehr S, Krasnow M, Bryant G, Hagen E. Origins of music in credible signaling. Behav Brain Sci. 2020;1-41.

16. Bando H. Recognition of happiness from subjective, psychological and neuroimaging points of view. $J$ Psychol Clin Psychiatry. 2020;11(2):56-58.

17. Sato W, Kochiyama T, Uono S, et al. Resting-state neural activity and connectivity associated with subjective happiness. Sci Rep. 2019;9(1):12098.

18. Li Y, Guan D, Yu Y, et al. A psychophysical measurement on subjective well-being and air pollution. Nature Communications. 2019;10(1).

19. Rahm T, Heise E. Teaching Happiness to Teachers - Development and Evaluation of Training in Subjective Well-Being. Front Psychol. 2019; 10:2703.

20. Cowen AS, Fang X, Sauter D, et al. What music makes us feel: At least 13 dimensions organize subjective experiences associated with music across different cultures. Proc Natl Acad Sci USA. 2020;117(4):1924 1934.
21. de Witte M, Spruit A, van Hooren S, et al. Effects of music interventions on stress-related outcomes: A systematic review and two meta-analyses. Health Psychol Rev. 2020;14(2):294-324.

22. Bainbridge CM, Bertolo M, Youngers J, et al. Infants relax in response to unfamiliar foreign lullabies. Nat Hum Behav. 2021;5(2):256-264.

23. de Witte M, da Silva Pinho A, Stams GJ, et al. Music therapy for stress reduction: a systematic review and meta-analysis. Health Psychol Rev. 2020;14(1):1-26.

24. Agres K, Schaefer R, Volk A, et al. Music, computing, and health: A roadmap for the current and future roles of music technology for healthcare and well-being. Music \& Science. 2021;4:1-32.

25. Bando H. Medical Progress from Bio-Psycho-Social Points of View Associated with Happiness of People. Biomed Sci J. 2020;1:101. 